PROCEEDINGS OF THE

AMERICAN MATHEMATICAL SOCIETY

Volume 134, Number 12, December 2006, Pages 3625-3627

S 0002-9939(06)08434-6

Article electronically published on June 15, 2006

\title{
A SHORT PROOF OF AN INEQUALITY OF LITTLEWOOD AND PALEY
}

\author{
MIROSLAV PAVLOVIĆ
}

(Communicated by Michael T. Lacey)

Abstract. A very short proof is given of the inequality

$$
\int_{|z|<1}|\nabla u(z)|^{p}(1-|z|)^{p-1} d x d y \leq C_{p}\left(\frac{1}{2 \pi} \int_{0}^{2 \pi}\left|f\left(e^{i t}\right)\right|^{p} d t-|u(0)|^{p}\right),
$$

where $p>2$, and $u$ is the Poisson integral of $f \in L^{p}(\partial \mathbb{D}), \mathbb{D}=\{z:|z|<1\}$.

Let $\mathbb{D}$ denote the open unit disk of the complex plane, and $\mathbb{T}=\partial \mathbb{D}$. The following theorem was proved by Littlewood and Paley in [2].

Theorem LP. If $f$ is a real valued function of class $L^{p}(\mathbb{T}), p>2$, and if $u$ is the Poisson integral of $f$, then

$$
\int_{\mathbb{D}}|\nabla u(z)|^{p}\left(1-|z|^{2}\right)^{p-1} d A(z) \leq C_{p}\|f\|_{p}^{p}
$$

where $C_{p}$ is a constant depending only on $p$ and

$$
\|f\|_{p}^{p}=\frac{1}{2 \pi} \int_{0}^{2 \pi}\left|f\left(e^{i t}\right)\right|^{p} d t
$$

Here $d A$ stands for the Lebesgue measure normalized so that $A(\mathbb{D})=1$.

This theorem can easily be proved by using the Riesz-Thorin interpolation theorem. In [3] Luecking gave an elementary but rather long proof based on the formula

$$
\|f\|_{p}^{p}-|u(0)|^{p}=\frac{\left(p^{2}-p\right)}{2} \int_{\mathbb{D}}|\nabla u|^{2}|u|^{p-2} \log \frac{1}{|z|} d A(z) .
$$

This formula, a consequence of the Green formula, was used by P. Stein [5] to prove the Riesz theorem on conjugate functions (see [1, p. 55). We also start from (2), and reduce to the case of positive harmonic functions, which satisfy the following inequality:

If $u$ is a positive harmonic function on $\mathbb{D}$, then

$$
|\nabla u(z)| \leq 2\left(1-|z|^{2}\right)^{-1} u(z) \quad(z \in \mathbb{D}) .
$$

This inequality is obtained by applying the special case $z=0$ to the function

$$
w \mapsto u\left(\frac{z-w}{1-\bar{z} w}\right) .
$$

In fact, we shall prove a slightly improved version of Theorem LP.

Received by the editors May 18, 2005 and, in revised form, July 11, 2005.

2000 Mathematics Subject Classification. Primary 46E15.

The author was supported by MNZŽS Grant, No. ON144010, Serbia.

(C)2006 American Mathematical Society 3625 
Theorem 1. If $f$ is a real valued function of class $L^{p}(\mathbb{T}), p>2$, and if $u$ is the Poisson integral of $f$, then

$$
\int_{\mathbb{D}}|\nabla u(z)|^{p}\left(1-|z|^{2}\right)^{p-1} d A(z) \leq C_{p}\left(\|f\|_{p}^{p}-|u(0)|^{p}\right),
$$

where $C_{p}$ is a constant depending only on $p$.

Proof. Let $f \in L^{p}(\mathbb{T}), p>2$. Let $u_{i}(i=1,2)$ denote the Poisson integral of $f_{i}$, where $f_{1}=\max (f, 0)$ and $f_{2}=\max (-f, 0)$. Then $u_{i} \geq 0, u=u_{1}-u_{2}$ and

$$
\|f\|_{p}^{p}=\left\|f_{1}\right\|_{p}^{p}+\left\|f_{2}\right\|_{p}^{p} .
$$

Also, since

$$
|\nabla u|^{p} \leq 2^{p-1}\left(\left|\nabla u_{1}\right|^{p}+\left|\nabla u_{2}\right|^{p}\right)
$$

the proof reduces to the case where $u>0$. Then it follows from (2), (3) and the inequality

$$
\log \frac{1}{|z|} \geq \frac{1-|z|^{2}}{2}
$$

that

$$
\begin{aligned}
\|f\|_{p}^{p}-|u(0)|^{p} & \geq \frac{p^{2}-p}{4} \int_{\mathbb{D}}|\nabla u|^{2} u^{p-2}\left(1-|z|^{2}\right) d A(z) \\
& \geq \frac{p^{2}-p}{4} \int_{\mathbb{D}}|\nabla u|^{2} 2^{2-p}|\nabla u|^{p-2}\left(1-|z|^{2}\right)^{p-1} d A(z) .
\end{aligned}
$$

This proves (1) for $f>0$ with $C_{p}=2^{p} /\left(p^{2}-p\right)$. If $f$ is arbitrary, then we use (5), (6) and the inequality

$$
|a-b|^{p} \leq a^{p}+b^{p} \quad(a \geq 0, b \geq 0)
$$

to get

$$
\begin{aligned}
\|f\|_{p}^{p}-|u(0)|^{p} & \geq\left\|f_{1}\right\|_{p}^{p}-\left|u_{1}(0)\right|^{p}+\left\|f_{2}\right\|_{p}^{p}-\left|u_{2}(0)\right|^{p} \\
& \geq\left(p^{2}-p\right) / 2^{p} \int_{\mathbb{D}}\left(\left|\nabla u_{1}\right|^{p}+\left|\nabla u_{1}\right|^{p}\right)(1-|z|)^{p-1} d A(z) \\
& \geq 2^{1-p}\left(p^{2}-p\right) / 2^{p} \int_{\mathbb{D}}|\nabla u|^{p}(1-|z|)^{p-1} d A(z) .
\end{aligned}
$$

Hence

$$
\int_{\mathbb{D}}|\nabla u|^{p}\left(1-|z|^{2}\right)^{p-1} d A(z) \leq C_{p}\left(\|f\|_{p}^{p}-|u(0)|^{p}\right)
$$

with

$$
C_{p}=2^{2 p-1} /\left(p^{2}-p\right)
$$

This completes the proof.

Remark 1. Inequality (4) can be written as

$$
\|f\|_{p}^{p}-|f(0)|^{p} \geq c_{p} \int_{\mathbb{D}}|\nabla u|^{p}\left(1-|z|^{2}\right)^{p-1} d A(z),
$$

which can be viewed as a refinement of the inequality $\|f\|_{p}^{p}-|f(0)|^{p} \geq 0$, a consequence of the subharmonicity of the function $|u(z)|^{p}$.

Remark 2. Inequality (4) holds for functions with values in a Hilbert space, but the proof is more delicate. See 4 . 


\section{REFERENCES}

1. P.L. Duren, Theory of $H^{p}$-spaces, Academic Press, New York and London, 1970. MR0268655 $(42: 3552)$

2. J.E. Littlewood and R.E.A.C. Paley, Theorems on Fourier series and power series. II, Proc. Lond. Math. Soc. 42(1936), 52-89.

3. D.H. Luecking, A new proof of an inequality of Littlewood and Paley, Proc. Amer. Math. Soc. 103(1988), 887-893. MR0947675 (89g:30067)

4. M. Pavlović, A Littlewood-Paley theorem for subharmonic functions with subharmonic Laplacian, Publ. Inst. Math. (Belgrade) 68(82)(2000), 77-82. MR1826098 (2002b:30040)

5. P. Stein, On a theorem of M. Riesz, J. London Math. Soc. 8(1933), 52-89.

MatematičKi Fakultet, Univerzitet u Beogradu, Studentski trg 16, 11000 Belgrade, Serbia, Yugoslavia

E-mail address: pavlovic@matf.bg.ac.yu 